\title{
Control MPC basado en un modelo LTV para un control de seguimiento de trayectoria
}

\author{
Sara Mata, Asier Zubizarreta, Ione Nieva, Itziar Cabanes, Charles Pinto \\ sara.matac@ehu.eus, asier.zubizarreta@ehu.eus, ione.nieva@gmail.com, itziar.cabanes@ehu.eus, charles.pinto@ehu.eus \\ Departamento de Ingeniería de Sistemas y Automática, \\ Escuela Superior de Ingeniería de Bilbao, UPV/EHU
}

\section{Resumen}

Este artículo presenta una aplicación de seguimiento de trayectoria para vehículos mediante Control Predictivo basado en Modelo (MPC) con un modelo Linealmente Variable en el Tiempo (LTV). El sistema de control está diseñado para garantizar un correcto seguimiento de la trayectoria bajo ciertos criterios de confort. Por lo tanto, no sólo se considera el error lateral, sino también el error de orientación respecto de la trayectoria objetivo. Además, también se consideran restricciones estrictas en la señal de control, en la variación de la señal de control y en el error lateral de seguimiento de la trayectoria. Se exponen resultados experimentales para diferentes tipos de trazados, concluyendo con un circuito. En todos los casos se considera un amplio rango de velocidades incluyendo elevadas velocidades.

Palabras clave: MPC, LTV, dinámica lateral

\section{INTRODUCCIÓN}

Durante las últimas décadas, el desarrollo tecnológico ha conllevado el incremento del número de dispositivos electronicos en los automóviles [9]. Hoy en día, los vehículos incluyen sofisticados sistemas de seguridad activa con el fin de evitar accidentes y al mismo tiempo ayudar al conductor con la controlabilidad y estabilidad del vehículo, especialmente en situaciones de emergencia [2].

Los primeros trabajos en sistemas de seguridad activa datan de la década de los 80 y estaban enfocados principalmente a la mejora del comportamiento longitudinal del vehículo. Ejemplos de estos sistemas son el Sistema Antibloqueo de Ruedas o Frenos Antibloqueo (ABS) que mejora la eficiencia de la frenada evitando el bloqueo de las ruedas al frenar; o el Control de Tracción (TC) que previene el deslizamiento de las ruedas y mejora la estabilidad del vehículo y la maniobrabilidad maximizando la tracción y las fuerzas laterales entre los neumáticos y la carretera. Estos sistemas han sido mejorados durante estos años, proponiendo diferentes sistemas de Control de Tracción, como el Programa de Estabilidad Electronica (ESP), entre otros.

Recientes progresos en la tecnología sensórica, como la visión por ordenador, la geolocalización y la reconstrucción de entornos 3D han impulsado las posibilidades en los sistemas de seguridad activa y han promovido la base para el diseño de sistemas de guiado avanzados para vehículos autonomos y semiautonomos [1], [19]. Estos sistemas aportan multiples ventajas en la estabilidad, seguridad y ergonomía de los vehículos: Sistema de Mantenimiento en Carril, que ayudan al conductor a mantener su vehículo dentro del carril y evitar salidas de calzada [4]; Sistemas de Cambio de Carril, para ayudar al conductor a hacer un adelantamiento de forma segura [15]; o Sistemas Anticolisión, que detectan un obstáculo en la calzada y ayudan al conductor a evitarlo con una nueva trazada pero de forma segura [21].

Estos sistemas de seguridad activa están normalmente basados en el uso de tres módulos: planificación de la trayectoria y localización, toma de decisión y ley de control. El módulo de planificación y localización aporta, por un lado tanto la localización exacta del vehículo en cada instante, como la futura trayectoria que debe seguir y/o los objetos que rodean al vehículo, como por ejemplo, las líneas de los carriles para Sistemas de Manteniemiento en el Carril [14]. El módulo de la ley de control está enfocado en el seguimiento de la trayectoria calculada por el planificador de una forma segura y óptima. Por último el módulo de toma de decisión usa la información proporcionada por el módulo de planificación de la trayectoria y localización para determinar estados de riesgo y reconducir el vehículo a una trayectoria segura [11], [18]. Este algoritmo debe estar apropiadamente ajustado como para que sus acciones no sean invasivas para el conductor en circustancias normales.

En la literatura, se pueden encontrar una gran variedad de trabajos relacionados con el control de seguimiento de trayectoria, donde se proponen trabajos con diversas estrategias de control. Por un lado, se emplean técnicas de control tradicionales para aplicaciones del seguimiento de trayectoria [12], [22]. También se pueden encontrar propuestas 
donde se emplean técnicas de control inteligente [15], [16], o incluso aplicaciones con Redes Neuronales [10]. Sin embargo, no considerar la dinámica del vehículo implica el incremento del error de seguimiento en ciertas maniobras.

Por otro lado, se pueden encontrar técnicas de control que sí consideran modelos de dinámica vehicular. Las técnicas de control robusto son comunmente empleadas debido a su robustez ante imprevistos y su capacidad de rechazar perturbaciones [5] [20]. Sin embargo, éstas son técnicas complejas y por lo tanto computacionalmente exigentes. Por su capacidad para gestionar sistemáticamente modelos no lineales y variables en el tiempo y operar cerca del límite de estados admisibles y entradas, el Control Predictivo basado en Modelo (MPC) ha sido ampliamente usado en los problemas de seguimiento de trayectoria [2]. Las aplicaciones implementadas utilizando MPC están principalmente enfocadas en la optimización del modelo [4] o del controlador [8], aunque también se pueden encontrar trabajos donde se consideran ambos problemas [7].

En este trabajo se propone un control MPC con coste computacional eficiente basado en un modelo bicicleta Linealmente Variable en el Tiempo (LTV) para el control de desplazamiento lateral. En la propuesta se optimizan tanto el desplazamiento lateral como el ángulo de guiñada, aportando un seguimiento de trayectoria más preciso. Además, el uso de un modelo LTV dependiente de la velocidad longitudinal aporta un rango operacional más amplio que el de otros trabajos. A su vez, se implementan estándares de confort imponiendo restricciones en la variación del ángulo de giro de las ruedas.

El resto del artículo se estructura de la siguiente forma: el modelo LTV usado para la predicción de la trayectoria se detalla en el Apartado 2; la metodología empleada para calcular la trayectoria en el horizonte de predicción se explica en el Apartado 3; el control MPC propuesto se presenta en el Apartado 4; los resultados de la simulación se discuten en la sección 5; y finalmente las conclusiones y futuros trabajos se detallan en el Apartado 6 .

\section{MODELO DEL VEHÍCULO}

Aunque la dinámica vehicular es generalmente engorrosa, el modelo comunmente conocido como modelo bicicleta se emplea generalmente para la aproximación de la dinámica lateral vehicular [17]. Este modelo considera dos grados de libertad que están representados por la posición lateral $y$, medida a lo largo del eje lateral del vehículo, y el ángulo de guiñada $\psi$, medido en el eje $z$ del vehículo, y tal y como se muestra en la Figura 1.

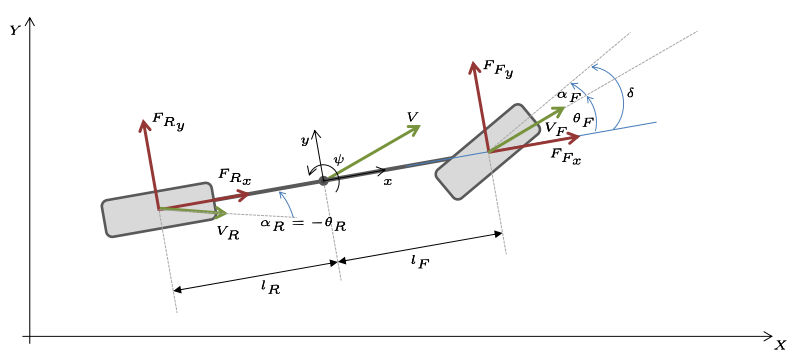

Figura 1: Diagrama del modelo bicicleta de dos grados de libertad

La dinámica lateral se calcula aplicando la segunda ley de Newton para las fuerzas en el eje $y$ y los momentos alrededor del eje $z$,

$$
\begin{aligned}
m a_{y} & =F_{F_{y}}+F_{R_{y}} \\
I_{z} \ddot{\psi} & =l_{F} F_{F_{y}}-l_{R} F_{R_{y}}
\end{aligned}
$$

donde $m$ es la masa del vehículo, $a_{y}$ es la aceleración lateral inercial del vehículo en el centro de gravedad (CoG) y en la dirección del eje $y$ del vehículo, $F_{F_{y}}$ y $F_{R_{y}}$ son las sumas de las fuerzas laterales en los neumáticos delanteros y traseros respectivamente, $I_{z}$ es el momento inercial alrededor del eje $z$ del vehículo, $\ddot{\psi}$ es la aceleración del ángulo de guiñada y $l_{F}$ y $l_{R}$ son las distacias desde el CoG hasta los ejes delantero y trasero respectivamente (Figura 1).

La aceleración lateral inercial $a_{y}$ se debe al movimiento en el eje $y$ y de la aceleración centrípeta $\dot{x} \dot{\psi}$, así que se puede calcular como [17]

$$
a_{y}=\ddot{y}+\dot{x} \dot{\psi}
$$

Resultados experimentales presentados en [17] demuestran que la fuerza lateral del neumático es proporcional al ángulo de deslizamiento, para pequeños ángulos de deslizamiento. De este modo, las fuerzas laterales de los neumáticos $F_{F_{y}}$ y $F_{R_{y}}$ se definen

$$
\begin{aligned}
& F_{F_{y}}=2 C_{\alpha_{F}} \alpha_{F} \\
& F_{R_{y}}=2 C_{\alpha_{R}} \alpha_{R}
\end{aligned}
$$

donde $F_{F_{y}}$ y $F_{R_{y}}$ son la suma de las fuerzas asociadas a los neumáticos derecho e izquiero en cada eje, $C_{\alpha_{F}}$ y $C_{\alpha_{R}}$ son la rigidez en curva para los neumáticos delanteros y traseros respectivamente y $\alpha_{F}$ y $\alpha_{R}$ son los ángulos de deslizamiento.

El ángulo de deslizamiento se define como el ángulo entre la orientación del neumático y la orientación del vector velocidad de la rueda. Por lo tanto, el ángulo de deslizamiento delantero $\alpha_{F}$ depende 
del ángulo de giro de las ruedas $(\delta)$, mientras que el ángulo de deslizamiento trasero $\alpha_{R}$ no.

$$
\begin{aligned}
& \alpha_{F}=\delta-\theta_{F} \\
& \alpha_{R}=-\theta_{R}
\end{aligned}
$$

donde $\theta_{F}$ y $\theta_{R}$ son los ángulos de los vectores de la velocidad respecto al eje longitudinal del vehículo,

$$
\begin{aligned}
\tan \theta_{F} & =\frac{\dot{y}+l_{F} \dot{\psi}}{\dot{x}} \\
\tan \theta_{R} & =\frac{\dot{y}-l_{R} \dot{\psi}}{\dot{x}}
\end{aligned}
$$

Combinando las Ecuaciones (1) a (5) y considerando la aproximación para ángulos pequeños, se puede definir el modelo de la dinámica vehicular en espacio de estados. Este modelo se representa mediante la variable del espacio de estados $\mathbf{x}_{\mathbf{c}}(t)=[y(t) \dot{y}(t) \psi(t) \dot{\psi}(t)]^{T}$ y donde la variable de control se corresponde con el ángulo de giro de las ruedas $u_{c}(t)=\delta(t)$.

$$
\begin{aligned}
& \dot{\mathbf{x}}_{\mathbf{c}}(t)=\mathbf{A}_{\mathbf{c}} \mathbf{x}_{\mathbf{c}}(t)+\mathbf{B}_{\mathbf{c}} u_{c}(t) \\
& \mathbf{y}_{\mathbf{c}}(t)=\mathbf{C}_{\mathbf{c}} \mathbf{x}_{\mathbf{c}}(t)
\end{aligned}
$$

La salida del sistema considera tanto el desplazamiento lateral como el ángulo de guiñada $\mathbf{y}_{\mathbf{c}}(t)=$ $[y(t) \psi(t)]^{T}$. Además, la posición del vehículo se mide en el eje delantero, lo que permite considerar un sistema de seguimiento de trayectoria más realista. Para este fin, el desplazamiento lateral se calcula en términos del ángulo de guiñada $\psi$,

$$
y_{F w h e e l}=y+l_{F} \psi
$$

Por lo tanto, las matrices $\mathbf{A}_{\mathbf{c}}, \mathbf{B}_{\mathbf{c}}$ and $\mathbf{C}_{\mathbf{c}}$ para el espacio de estados están representadas por,

$$
\mathbf{A}_{\mathbf{c}}=\left[\begin{array}{cccc}
0 & 1 & 0 & 0 \\
0 & a_{c_{1}} & 0 & a_{c_{2}} \\
0 & 0 & 0 & 1 \\
0 & a_{c_{3}} & 0 & a_{c_{4}}
\end{array}\right]
$$

donde,

$$
\begin{aligned}
a_{c_{1}} & =-\frac{2 C_{\alpha F}+2 C_{\alpha R}}{m \dot{x}} \\
a_{c_{2}} & =-\frac{2 l_{F} C_{\alpha F}-2 l_{R} C_{\alpha R}}{m \dot{x}}-\dot{x} \\
a_{c_{3}} & =-\frac{2 l_{F} C_{\alpha F}-2 l_{R} C_{\alpha R}}{I_{z} \dot{x}} \\
a_{c_{4}} & =-\frac{2 l_{F}^{2} C_{\alpha F}+2 l_{R}^{2} C_{\alpha R}}{I_{z} \dot{x}}
\end{aligned}
$$

$$
\begin{gathered}
\mathbf{B}_{\mathbf{c}}=\left[\begin{array}{c}
0 \\
\frac{2 C_{\alpha F}}{m} \\
0 \\
\frac{2 l_{F} C_{\alpha F}}{I_{z}}
\end{array}\right] \\
\mathbf{C}_{\mathbf{c}}=\left[\begin{array}{llll}
1 & 0 & l_{F} & 0 \\
0 & 0 & 1 & 0
\end{array}\right]
\end{gathered}
$$

\section{TRAYECTORIA DE REFERENCIA}

El desarrollo del MPC requiere el previo conocimiento y definición de la trayectoria de referencia. En este trabajo, la trayectoria de referencia se define como una serie de coordenadas en el sistema de coordenadas inercial $\left(X_{i}, Y_{i}\right)$. Esta trayectoria define la posición central del vehículo y está suministrada por un sistema generador de trayectoria externo.

Para procesar la trayectoria, se define una nueva variable, Station $\left(S_{i}\right)$, que representa la distancia longitudinal cubierta a lo largo de la trayectoria deseada y se calcula para cada par de coordenadas (Figura 2). Esta variable permite definir únicamente cualquier posición de la carretera, ya que para cada valor de esta variable sólo existe un par de coordenadas $\left(X_{i}, Y_{i}\right)$ que define esta posición en el sistema de referencia global.

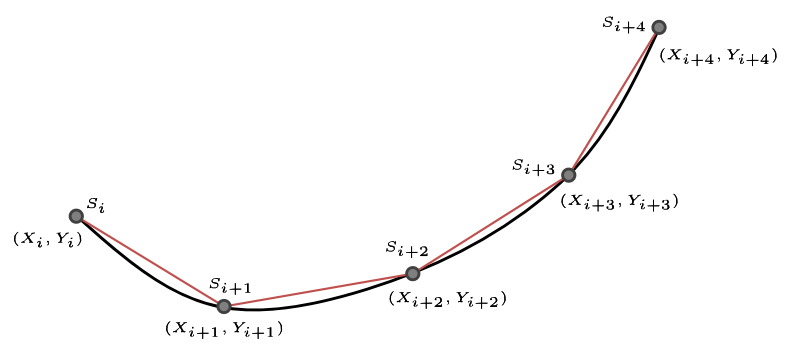

Figura 2: Definición de la trayectoria de referencia que debe seguir el vehículo

El valor actual de Station se calcula como la suma de su valor anterior y la distancia hasta el nuevo punto. Con el fin de simplificar su cálculo, se emplea una interpolacion lineal (Figura 2). Así, todos los puntos situados en la perpendicular a la trayectoria tienen el mismo valor de Station $\left(S_{i}\right)$,

$$
S_{i}=S_{i-1}+\sqrt{\left(x_{i}-x_{i-1}\right)^{2}+\left(y_{i}-y_{i-1}\right)^{2}}
$$

El procedimiento para calcular la trayectoria de referencia o trayectoria objetivo se resume en la Figura 3. Para una posición dada del CoG 
$\left(x_{C G}, y_{C G}\right)$, se calcula el punto en la trayectoria de referencia que está a la mínima distancia $\left(x_{m i n}, y_{m i n}\right)$. Este punto está en la normal a la trayectoria y que cruza el CoG. Así, se puede obtener el valor correspondiente de Station $\left(S_{m i n}\right)$. A continuación se calculan los siguientes valores de Station, que son necesarios para la ley de control del MPC.

$\mathbf{S}_{\text {target }}=\left[S_{m i n}+T_{s} \dot{x}, S_{m i n}+2 T_{s} \dot{x}, \ldots, S_{m i n}+H_{p} T_{s} \dot{x}\right]$

donde $\dot{x}$ es la velocidad longitudinal del vehículo, $H_{p}$ es el horizonte de predicción y $T_{s}$ es el tiempo de muestreo. Dados los siguiente valores $S_{\text {target }}$, se pueden obtener las coordinadas $x$ e $y$ de los puntos objetivo $\left(\mathbf{x}_{\text {target }}, \mathbf{y}_{\text {target }}\right)$ donde

$$
\begin{aligned}
& \mathbf{x}_{\text {target }}=\left[x_{\text {target }_{1}}, x_{\text {target }_{2}}, \ldots, x_{\text {target }_{H_{p}}}\right] \\
& \mathbf{y}_{\text {target }}=\left[y_{\text {target }_{1}}, y_{\text {target }_{2}}, \ldots, y_{\text {target }_{H_{p}}}\right]
\end{aligned}
$$

Finalmente, se definen las futuras referencias para la ley de control. Las variables que se minimizan en la ley de control son el desplazamiento lateral $\left(e_{x_{i}}=x_{\text {target }_{i}}-x_{c g}, e_{y_{i}}=y_{\text {target }_{i}}-y_{c g}\right.$ donde $\left.i=1,2 . . H_{p}\right)$, transformado del sistema de coordenadas global al local, y la orientación respecto a la trayectoria de referencia, que se calcula como la pendiente entre dos puntos consecutivos $\left(m_{1}\right.$, $\left.m_{2}, \ldots m_{H_{p}}\right)$.

$$
\mathbf{w}=\left[\begin{array}{c}
e_{x_{1}} \cos (\psi)-e_{y_{1}} \sin (\psi) \\
\arctan \left[m_{1}\right] \\
e_{x_{2}} \cos (\psi)-e_{y_{2}} \sin (\psi) \\
\arctan \left[m_{2}\right] \\
\cdots \\
e_{x_{H_{p}}} \cos (\psi)-e_{y_{H_{p}}} \sin (\psi) \\
\arctan \left[m_{H_{p}}\right]
\end{array}\right]
$$

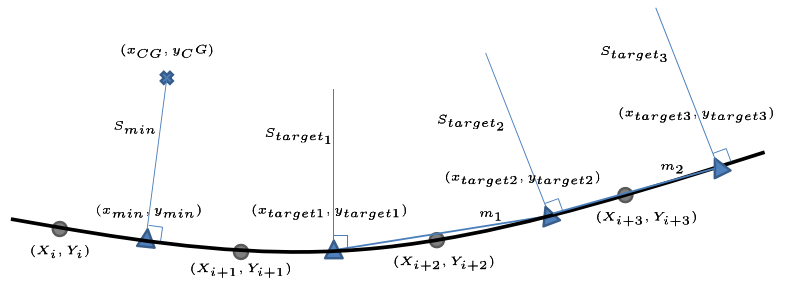

Figura 3: Definición de los puntos de la trayectoria de referencia

\section{CONTROL}

Se propone un sistema de seguimiento de trayectoria basado en un control MPC para el guiado lateral del vehículo. El principal objetivo del controlador es minimizar tanto el desplazamiento lateral como la desviación del ángulo de guiñada del vehículo mediante la aplicación del ángulo óptimo de giro de las ruedas $(\delta)$ para el seguimiento de la trayectoria de referencia de forma óptima. La base de los enfoques MPC es el uso de un horizonte de predicción $\left(H_{p}\right)$, en el cual se optimiza una función de coste empleando el modelo de predicción [3].

Debido a su capacidad para gestionar sistemáticamente modelos no lineales y variables en el tiempo, así como con restricciones y operar cerca de los límides de los estados admisibles y de las entradas, el MPC ha sido ampliamente usado en los problemas de seguimiento de trayectoria [2]. La propuesta se basa en un modelo LTV que permite una ejecución rápida y mantiene la precisión de seguimiento de trayectoria requerida.

\subsection{Modelo de predicción}

El control MPC se desarrolla para tiempo discreto, por lo que el modelo de vehículo presentado en la Ecuación (6) se discretiza con el Retenedor de Orden Cero $(\mathrm{ZOH})$ y se representa mediante la formulación del espacio de estados.

$$
\begin{aligned}
\mathbf{x}_{\mathbf{d}}(k+1) & =\mathbf{A}_{\mathbf{d}} \mathbf{x}_{\mathbf{d}}(k)+\mathbf{B}_{\mathbf{d}} u_{d}(k) \\
\mathbf{y}_{\mathbf{d}}(k) & =\mathbf{C}_{\mathbf{d}} \mathbf{x}_{\mathbf{d}}(k)
\end{aligned}
$$

donde $\mathbf{x}_{\mathbf{d}}(k), u_{d}(k)$ y $\mathbf{y}_{\mathbf{d}}(k)$ son el estado del vehículo, la señal de control y la salida del vehículo y $\mathbf{A}_{\mathbf{d}}, \mathbf{B}_{\mathbf{d}}, \mathbf{C}_{\mathbf{d}}$ son las matrices discretizadas del espacio de estados de las Ecuaciones (8),(9) y (10) respectivamente.

Usando el modelo de predicción de la Ecuación (15) se puede predecir la trayectoria futura $\hat{\mathbf{y}} \mathrm{du}-$ rante el horizonte de predicción $H_{p}$. Con el fin de mejorar la precisión en la predicción de la trayectoria, el modelo de predicción se formula como un modelo linealmente variable en el tiempo (LTV), que emplea la velocidad del vehículo en el instante inicial de la predicción y se asume constante durante todo el horizonte de predicción en aras del coste computacional.

\subsection{Función de coste}

La función de coste propuesta $J\left(H_{p}, H_{c}\right)$ penaliza las desviaciones respecto de la trayectoria de referencia deseada y la desviación del ángulo de guiñada deseado.

$J\left(H_{p}, H_{c}\right)=(\hat{\mathbf{y}}-\mathbf{w})^{T} \mathbf{Q}(\hat{\mathbf{y}}-\mathbf{w})+\left(\Delta \mathbf{u}^{T} \mathbf{R} \Delta \mathbf{u}\right)$ 
donde $\mathbf{w}$ define las referencias futuras del sistema para cada instante de muestreo en el horizonte de predicción (Ecuación (14)), $\Delta \mathbf{u}$ es la señal de control que se desea calcular (variación del ángulo de giro de las ruedas) y $\mathbf{Q}=\operatorname{diag}(\mathbf{q})$ y $\mathbf{R}=\operatorname{diag}(\mathbf{r})$ son las correspondientes matrices de ponderación del error de seguimiento y de la señal de control.

\subsection{Ley de control}

La ley de control del MPC se deriva de la optimización de la función de coste de Ecuación (16), que incluye el modelo de predicción. Así, es necesario minimizar la función de coste $J$ para obtener los valores óptimos de las señales de control incrementales $\boldsymbol{\Delta} \mathbf{u}^{*}$ que permiten al vehículo minimizar el error de seguimiento de trayectoria,

$$
\begin{aligned}
& \boldsymbol{\Delta} \mathbf{u}^{*}=\min J(\hat{\mathbf{y}}-\mathbf{w}, \boldsymbol{\Delta} \mathbf{u}) \\
& \text { s.t. } \\
& \underline{u} \leq u(k) \leq \bar{u} \\
& \underline{\Delta u} \leq \Delta u(k) \leq \overline{\Delta u} \\
& \underline{\mathbf{y}} \leq \mathbf{y}(k) \leq \overline{\mathbf{y}}
\end{aligned}
$$

donde se consideran restricciones en el ángulo de giro de las ruedas $(u(k))$, la variación del ángulo $(\Delta u(k))$ y el desvio $(\mathbf{y}(k))$ con el fin de reproducir un comportamiento más realista del vehículo.

Una vez calculada la secuencia, sólo se aplica el primer instante de la señal óptima predicha, la correspondiente al instante actual,

$$
u(k)=\Delta u(k)+u(k-1)
$$

\section{RESULTADOS}

El controlador MPC y el generador de trayectoria de referencia se han implementado para realizar un seguimiento de trayectoria a diferentes velocidades y para diferentes tipos de curvatura. La señal de control es el ángulo de giro de las ruedas delanteras $(u=\delta)$ y el objetivo es seguir la trayectoria lo mejor posible minimizando la desviación del vehículo respecto a la trayectoria de referencia.

El MPC propuesto ha sido validado implementándolo mediante el software MATLAB/Simulink [13]. El generador de trayectoria de referencia aporta al controlador MPC las futuras referencias de posición y orientación (w) detalladas en la Ecuación (14), considerando una trayectoria conocida. Dadas las referencias futuras $(\mathbf{w})$ y considerando la posición y orientación actuales $(\mathbf{x})$ y las velocidades $(\dot{\mathbf{x}})$, el controlador MPC calcula la señal de control, que es el ángulo de giro de las ruedas $(u=\delta)$, que se debe aplicar en el vehículo (Figura 4).

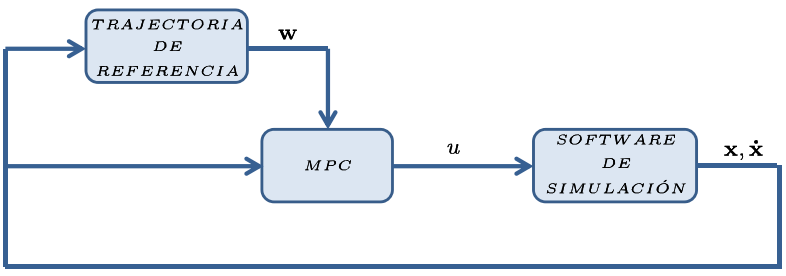

Figura 4: Diagrama del control MPC propuesto

Con el fin de simular la dinámica vehicular, se emplea el software Carmaker, de IPG Automotive [6], . El software de simulación provee de la posición y orientación del CoG del vehículo y su velocidad ( $\mathrm{x}$ y $\dot{\mathrm{x}}$ ). Los parámetros principales del vehículo se recogen en la Tabla 1.

Tabla 1: Parámetros del vehículo IPG Carmaker

\begin{tabular}{l|l}
\multicolumn{1}{c}{ Parameter } & Value \\
\hline$m:$ masa del vehículo & $1412 \mathrm{~kg}$ \\
\hline$l_{F}:$ distancia CoG - eje delantero & $1.016 \mathrm{~m}$ \\
\hline$l_{R}:$ distancia CoG - eje trasero & $1.564 \mathrm{~m}$ \\
\hline$I_{z}:$ inercia del ángulo de guiñada & $1536.7 \mathrm{~kg} \mathrm{~m}{ }^{2}$ \\
\hline$c_{\alpha F}:$ rigidez en curva eje delantero & $956.08 \mathrm{~N} / \mathrm{rad}$ \\
\hline$c_{\alpha R}:$ rigidez en curva eje trasero & $956.08 \mathrm{~N} / \mathrm{rad}$
\end{tabular}

Las simulaciones se han llevado a cabo escogiendo un amplio rango de velocidades comunes $(30,50,80,100,120 \mathrm{~km} / \mathrm{h})$ y tres tipos de trayectorias: una curva abierta, una curva larga y cerrada y un circuito que combina diferentes tipos de trazados. El modelo de vehículo de Dynacar incluye un conductor virtual, que conduce el vehículo considerando la trayectoria. Este conductor virtual se ha configurado para que alcance una aceleración longitudinal y lateral máxima de $0,3 \mathrm{~g}$ $\left(\approx 2,94 \mathrm{~m} / \mathrm{s}^{2}\right)$, que es el límite de una conducción confortable.

Se ha empleado un periodo de muestreo de $T_{s}=$ $1,5 s$, que para una velocidad de travesía de $30 \mathrm{~km} / \mathrm{h}$ cubre $12,5 \mathrm{~m}$ y para una de $120 \mathrm{~km} / \mathrm{h}$ $50 \mathrm{~m}$, y donde el horizonte de predicción es $H_{p}=$ 20 y el horizonte de control $H_{c}=10$. Las matrices de ponderación sintonizadas experimentalmente son $Q=\operatorname{diag}(500,75)$ and $R=1$. La restricción de la señal de control viene dada por la estructura del vehículo del modelo y la señal de control incremental por aspectos de confort. La restricción de salida cubre el desplazamiento lateral, que corresponde al error lateral, y al ángulo de guiñada. Así, considerando una carretera estándar de $3,5 \mathrm{~m}$ de ancho y un vehículo de $2 m$ de ancho, se establece un valor máximo de $0,6 \mathrm{~m}$ de desvío para que el vehículo no se salga de su carril. 


$$
\begin{array}{r}
-0,7156 \mathrm{rad} \leq u(k) \leq 0,7156 \mathrm{rad} \\
-0,0017 \mathrm{rad} / T_{s} \leq \Delta u(k) \leq 0,0017 \mathrm{rad} / T_{s} \\
(-0,6 \mathrm{~m},-\pi \mathrm{rad}) \leq \mathbf{y}(k) \leq(0,6 \mathrm{~m}, \pi \mathrm{rad})
\end{array}
$$
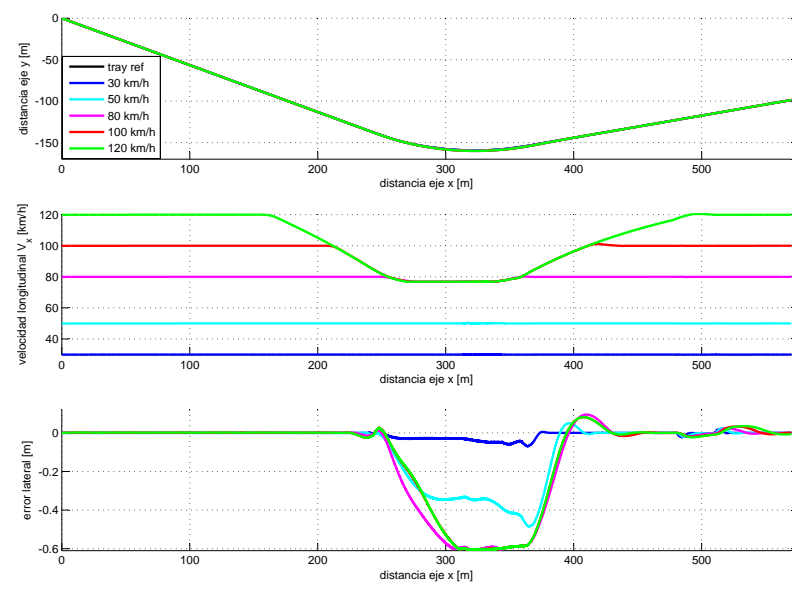

Figura 5: Simulación para una curva abierta

Se han llevado a cabo tres experimentos. El primer experimento considera al vehículo circulando por una curva abierta que se puede conducir a altas velocidades sin superar la aceleración lateral máxima de $0,3 \mathrm{~g}$. Los resultados de este primer experimento se muestran en Figura 5 para diferentes velocidades longitudinales. Nótese que el modelo de conductor virtual implementado en el modelo del vehículo adapta la velocidad del vehículo en función de la trayectoria, considerando los límites de aceleración fijados, por lo que reduce la velocidad hasta un valor aceptale $(76,84 \mathrm{~km} / \mathrm{h})$ para realizar el giro. Como se observa, la trayectoria se sigue adecuadamente para todo el rango de velocidades. Para la velocidad más baja de $30 \mathrm{~km} / \mathrm{h}$, el error lateral máximo entre la trayectoria de referencia y la trayectoria realizada es de 0,068 $\mathrm{m}$, la cual es despreciable. Sin embargo, para las velocidades más elevadas, el error máximo asciende a 0,6 $m$ durante el giro, el cual es el valor máximo, limitado por las restricciones.

Para este primer experimento, también se estudia el efecto de las restricciones. La diferencia entre el control MPC con y sin restricciones se representa en la Figura 6. La restricción de la señal de control $(u=\delta)$ es una restricción de diseño y aunque en este caso no se exceda es necesaria. Sin embargo, la restricción del valor incremental de la señal de control $(\Delta u=\Delta \delta)$ es una restricción de confort, para que el ángulo de giro de las ruedas no oscile. Como se puede observar en la Figura 6a, para $30 \mathrm{~km} / \mathrm{h}$ con esta restricción el ángulo de giro de las ruedas varía de forma más suave. Esto significa una conducción más realista, ya que la velocidad de giro y oscilaciones están limitadas.
Para la restricción de salida, su efecto se observa mejor a altas velocidades $(120 \mathrm{~km} / \mathrm{h}$ ) (Figura 6b). Sin ningun tipo de restricción, el vehículo sigue la trayectoria con un error despreciable, pero la señal de control estaría fuera de rango. Si se consideran las restricciones a la entrada, el error lateral asciende a 1,1 m. Éste es un error inaceptable, ya que significaría que el vehículo se sale de su carril. Por tanto, la restricción de salida es necesaria para garantizar que el vehículo permanece en su carril. Como las restricciones de salida requieren una estimación adecuada de la dinámica del modelo, esto demuesra que el modelo bicicleta LTV desarrollado es lo suficientemente preciso como para garantizar un adecuado seguimiento de trayectoria.

El segundo experimento es similar al primero, pero en este caso se ha considerado una curva cerrada y más larga. Como se representa en la Figura 7, con el fin de seguir adecuadamente la trayectoria, la velocidad longitudinal se reduce progresivamente hasta los $54 \mathrm{~km} / \mathrm{h}$ y se mantiene durante el giro. En este caso, el error lateral es menor que en el experimento previo, ya que el error máximo a altas velocidades es de $0,56 \mathrm{~m}$, y muy similar para todas las velocidades. Esto se debe a que la curva se toma a una velocidad longitudinal inferior que el caso anterior. Además, se puede observar que gracias al horizonte de predicción definido, el controlador MPC comienza a adaptarse al trazado antes del inicio de la curva, demostrando la adaptabilidad del controlador propuesto.
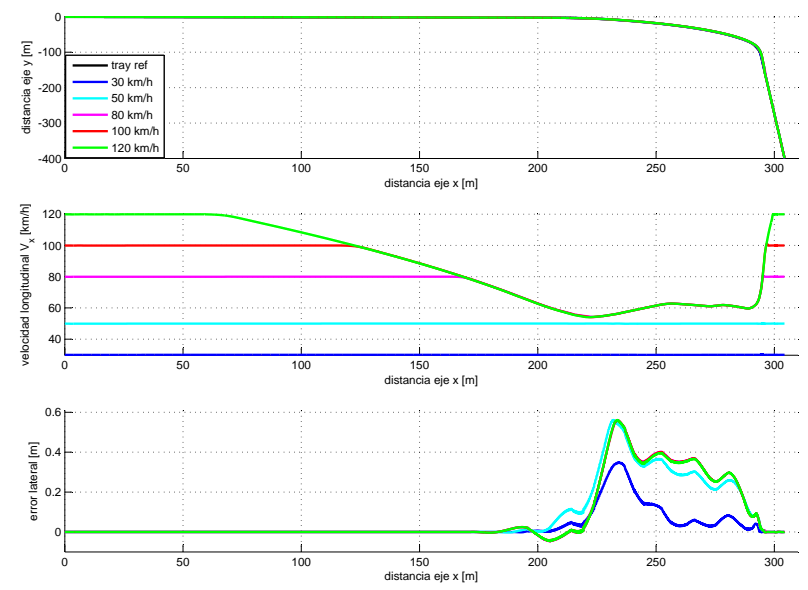

Figura 7: Simulación para una curva cerrada

Por último, el controlador MPC se ha validado en un circuito compuesto por diferentes tipos de curvas, desde muy cerradas o zig-zags hasta a trazados rectos. Los resultados se ilustran en la Figura 8 para una vuelta. El perfil de la velocidad longitudinal muestra cómo la velocidad del vehículo decrece al entrar en una curva y crece al salir de la misma. La velocidad máxima de $113 \mathrm{~km} / \mathrm{h}$ se 

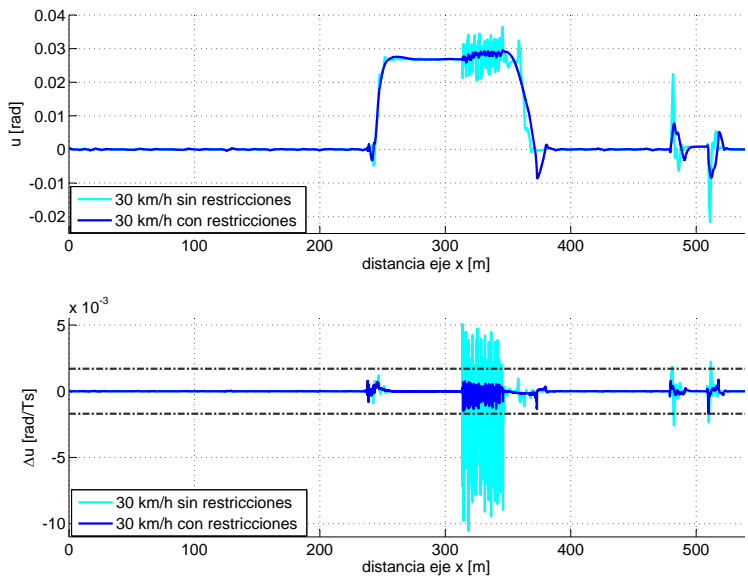

(a)

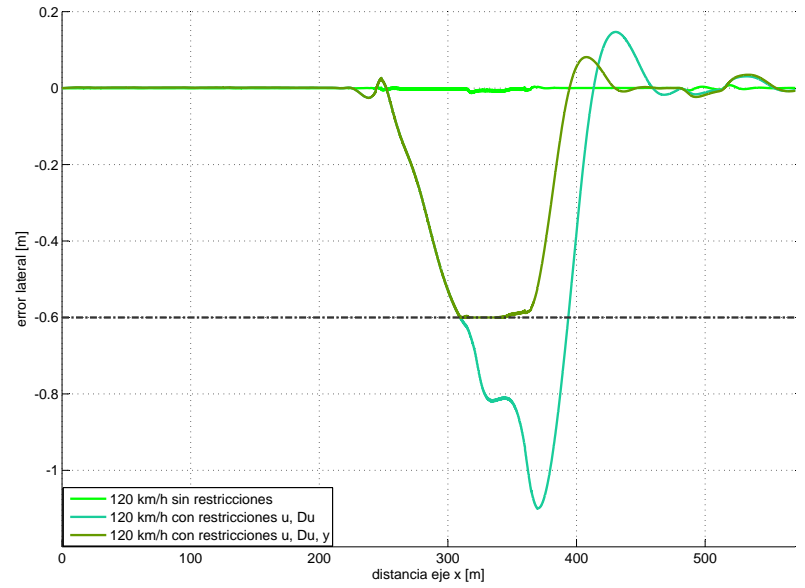

(b)

Figura 6: Uso de las restricciones para una curva abierta (30 and $120 \mathrm{~km} / \mathrm{h}$ )
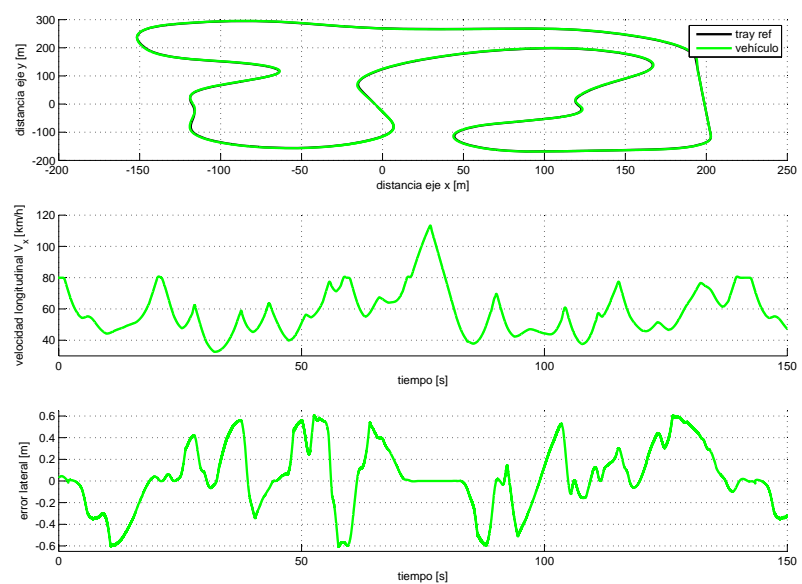

Figura 8: Simulación para una vuelta en un circuito

alcanza en la parte recta del circuito. Como se puede observar, el error lateral varía a lo largo del circuito, pero dentro de los límites fijados pos las restricciones. Así, el MPC desarrollado es capaz de predecir la trayectoria a seguir por el vehículo y es capaz de restringir su movimiento lateral, con el fin de que el vehículo se mantenga en su carril. Los resultados demuestran la efectividad de la propuesta.

\section{CONCLUSIONES}

En el presente trabajo se propone una aplicación de seguimiento de trayectoria empleado MPC con un modelo de predicción LTV. El enfoque propuesto optimiza tanto el desplazamiento lateral como el ángulo de guiñada obteniendo así un mejor seguimiento de trayectoria. El modelo de predicción LTV dependiente de la velocidad longitudinal, ofrece un mayor rango operacional para la aplicación. Los requerimientos de confort están impuestos por restricciones en el ángulo de giro de las ruedas y en su variación y por la consideración de la orientación en el MPC. Además, las restricciones de salida están consideradas en el error lateral para garantizar un correcto seguimiento de la trayectoria sin salirse de la calzada.

El control propuesto ha sido validado para un amplio rango de velocidades en tres experimentos diferentes. El primer y segundo experimento consisten en la validación del MPC mediante una curva abierta a elevadas velocidades y una más cerrada a menores velocidades. El tercer y último experimento consiste en un circuito con diferentes tipos de trazado. El vehículo circula por el circuito a una velocidad variable desde los $33 \mathrm{~km} / \mathrm{h}$ hasta los $113 \mathrm{~km} / \mathrm{h}$ y manteniendo el error lateral dentro de los límites.

Como futuros trabajos se considera el estudio de la estabilidad del MPC propuesto y la incorporación de consideraciones de robustez a la aplicación, para la compesación de perturbaciones.

\section{Agradecimientos}

Este trabajo ha sido parcialmente financiado por el Programa Predoctoral de Formación de Personal Investigador No Doctor BFI- 2012- 223 del Gobierno Vasco, el Ministerio de Economía y Competitividad MINECO\&FEDER en el marco del proyecto DPI- 2012-32882, así como por el proyecto UFI11/28 de la UPV/EHU.

\section{Referencias}

[1] M. Bertozzi, A. Broggi, and A. Fascioli. Vision-based intelligent vehicles: State of the art and perspectives. Robotics and Autono- 
mous Systems, 32(1):1 - 16, 2000.

[2] F. Borrelli, P. Falcone, T. Keviczky, J. Asgari, and D. Hrovat. MPC-based approach to active steering for autonomous vehicle systems. International Journal of Vehicle Autonomous Systems, 3(2-4):265-291, 2005.

[3] E. F. Camacho and C. Bordons. Model predictive control in the process industry. Springer Science \& Business Media, 2012.

[4] B.-C. Chen, B.-C. Luan, and K. Lee. Design of lane keeping system using adaptive model predictive control. IEEE International Conference on Automation Science and Engineering (CASE), pages 922-926, 2014.

[5] S. Hima, B. Lusseti, B. Vanholme, S. Glaser, and S. Mammar. Trajectory tracking for highly automated passenger vehicles. International Federation of Automatic Control (IFAC) World Congress, pages 12958-12963, 2011.

[6] IPG Automotive. CarMaker. http://ipg.de/, June 2016.

[7] E. Kayacan, E. Kayacan, H. Ramon, and W. Saeys. Distributed nonlinear model predictive control of an autonomous tractor and trailer system. Mechatronics, 24(8):926 - 933, 2014.

[8] B.-A. Kim, S.-H. Lee, Y. O. Lee, and C. C. Chung. Comparative study of approximate, proximate, and fast model predictive control with applications to autonomous vehicles. 12th International Conference on Control, Automation and Systems (ICCAS), pages 479-484, 2012.

[9] G. Leen and D. Heffernan. Expanding automotive electronic systems. Computer, 35(1):88-93, 2002.

[10] C. MacAdam, Z. Bareket, P. Fancher, and R. Ervin. Using neural networks to identify driving style and headway control behavior of drivers. Vehicle System Dynamics, 29(S1):143-160, 1998.

[11] S. Mammar. Driver co-pilote for vehicle lateral control at handling limits. IEEE 12th International Conference on Networking, Sensing and Control (ICNSC), pages 474-479, 2015 .

[12] R. Marino, S. Scalzi, G. Orlando, and M. Netto. A nested PID steering control for lane keeping in vision based autonomous vehicles. American Control Conference (ACC), pages 2885-2890, 2009.
[13] MathWorks. Matlab/Simulink. http://es.mathworks.com/, June 2016.

[14] J. McCall and M. Trivedi. Video-based lane estimation and tracking for driver assistance: Survey, system, and evaluation. IEEE Transactions on Intelligent Transportation Systems, 7(1):20-37, 2006.

[15] J. Naranjo, C. González, R. García, and T. D. Pedro. Lane-change fuzzy control in autonomous vehicles for the overtaking maneuver. IEEE Transactions on Intelligent Transportation Systems, 9(3):438-450, Sept 2008.

[16] J. E. Naranjo, C. Gonzalez, R. Garcia, T. de Pedro, and R. E. Haber. Power-steering control architecture for automatic driving. IEEE Transactions on Intelligent Transportation Systems, 6(4):406-415, Dec 2005.

[17] R. Rajamani. Vehicle Dynamics and Control. Springer, 2012.

[18] C. Sentouh, B. Soualmi, J. C. Popieul, and S. Debernard. Cooperative steering assist control system. IEEE International Conference on Systems, Man, and Cybernetics (SMC), pages 941-946, 2013.

[19] Z. Sun, G. Bebis, and R. Miller. On-road vehicle detection: A review. IEEE Transactions on Patter, 28(5):694-711, May 2006.

[20] G. Tagne, R. Talj, and A. Charara. Immersion and Invariance vs Sliding Mode Control for reference trajectory tracking of autonomous vehicles. European Control Conference (ECC), pages 2888-2893, 2014.

[21] V. Turri, A. Carvalho, H. E. Tseng, K. H. Johansson, and F. Borrelli. Linear model predictive control for lane keeping and obstacle avoidance on low curvature roads. 16th International IEEE Conference on Intelligent Transportation Systems (ITSC), pages 378383, 2013.

[22] P. Zhao, J. Chen, T. Mei, and H. Liang. Dynamic motion planning for autonomous vehicle in unknown environments. IEEE Intelligent Vehicles Symposium (IV), pages 284289, 2011. 